\title{
Comparison of Imaging Strategies with Conditional versus Immediate Contrast-Enhanced Computed Tomography in Patients with Clinical Suspicion of Acute Appendicitis
}

\author{
J. J. Atema • S. L. Gans • A. Van Randen • W. Laméris • H. W. van Es • \\ J. P. M. van Heesewijk • B. van Ramshorst • W. H. Bouma • W. ten Hove • \\ E. M. van Keulen • M. G. W. Dijkgraaf • P. M. M. Bossuyt • J. Stoker • \\ M. A. Boermeester
}

Received: 30 September 2014 / Revised: 12 December 2014 / Accepted: 30 January 2015 / Published online: 24 April 2015

(C) The Author(s) 2015. This article is published with open access at Springerlink.com

\begin{abstract}
Objectives To compare the diagnostic accuracy of conditional computed tomography (CT), i.e. CT when initial ultrasound findings are negative or inconclusive, and immediate CT for patients with suspected appendicitis.

Methods Data were collected within a prospective diagnostic accuracy study on imaging in adults with acute abdominal pain. All patients underwent ultrasound and CT, read by different observers who were blinded from the other modality. Only patients with clinical suspicion of appendicitis were included. An expert panel assigned a final diagnosis to each patient after 6 months of follow-up (clinical reference standard).

Results A total of 422 patients were included with final diagnosis appendicitis in 251 (60 \%). For 199 patients (47\%), ultrasound findings were inconclusive or negative. Conditional CT imaging correctly identified 241 of 251 (96\%) appendicitis cases (95\%CI, $92 \%$ to $98 \%$ ), versus
\end{abstract}

J. J. Atema $(\bowtie) \cdot$ S. L. Gans • W. Laméris · M. A. Boermeester Department of Surgery (G4-142), Academic Medical Centre, 1105 AZ Amsterdam, Netherlands

e-mail: j.j.atema@amc.nl

A. Van Randen · J. Stoker

Department of Radiology, Academic Medical Centre,

Amsterdam, Netherlands

W. Laméris

Department of Surgery, Spaarne Hospital, Hoofddorp, Netherlands

H. W. van Es · J. P. M. van Heesewijk

Department of Radiology, St Antonius Hospital,

Nieuwegein, Netherlands

B. van Ramshorst

Department of Surgery, St Antonius Hospital,

Nieuwegein, Netherlands
238 (95\%) with immediate CT (95\%CI, $91 \%$ to $97 \%)$. The specificity of conditional CT imaging was lower: $77 \%$ (95\%CI, $70 \%$ to $83 \%$ ) versus $87 \%$ for immediate CT ( $95 \% \mathrm{CI}, 81 \%$ to $91 \%$ ).

Conclusion A conditional CT strategy correctly identifies as many patients with appendicitis as an immediate CT strategy, and can halve the number of CTs needed. However, conditional CT imaging results in more false positives.

Key Points

- Conditional CT (CT after negative/inconclusive ultrasound findings) can be used for suspected appendicitis.

- Half the number of CT examinations is needed with a conditional strategy.

- Conditional CT correctly identifies as many patients with appendicitis as immediate CT.

- Conditional imaging results in more false positive appendicitis cases.

W. H. Bouma

Department of Surgery, Gelre Hospital, Apeldoorn, Netherlands

W. ten Hove

Department of Radiology, Gelre Hospital, Apeldoorn, Netherlands

E. M. van Keulen

Department of Radiology, Tergooi Hospital, Hilversum, Netherlands

M. G. W. Dijkgraaf

Clinical Research Unit, Academic Medical Centre,

Amsterdam, Netherlands

P. M. M. Bossuyt

Department of Clinical Epidemiology, Biostatistics, and

Bioinformatics, Academic Medical Center, Amsterdam, Netherlands 
Keywords Appendicitis · Diagnostic Imaging · Ultrasound · Tomography, X-Ray Computed · Predictive Value of Tests

\author{
Abbreviations \\ CT Computed tomography
}

with clinical suspicion of acute appendicitis. The second aim was to evaluate the diagnostic accuracy for other urgent surgical conditions in patients clinically suspected of acute appendicitis, since in clinical practice, also the correct identification of alternate underlying conditions is important.

\section{Methods}

Data for the present study were collected as part of a prospective, multi-centre, diagnostic accuracy study investigating the additional value of imaging on top of clinical evaluation in adult patients presenting with acute abdominal pain at the emergency department between March 2005 and November 2006. Details on the patient identification and imaging protocols have been published elsewhere $[6,12]$. The medical ethics committee approved the original study, and all included patients gave written informed consent. All included patients underwent ultrasound as well as CT within a few hours after presentation. Two different observers who were blinded from all other imaging findings independently evaluated the results of ultrasound and CT.

\section{Ultrasound}

All standardized ultrasound examinations were performed using a curved $3.5-5.0 \mathrm{MHz}$ array and a linear $10 \mathrm{MHz}$ array, and findings were recorded in a digital case record form. The following appendiceal characteristics were evaluated: complete visualization of the appendix (visualized from to base to the tip of the appendix), local transducer tenderness, compressibility of the appendix, a thickened appendix (diameter greater than $6 \mathrm{~mm}$ ), the presence of an appendicolith, an intact layered wall structure, periappendiceal fat infiltration, and free fluid adjacent to the appendix. Finally, an ultrasound diagnosis was selected from a predefined list of diagnoses [12]. The diagnosis of acute appendicitis was left to the discretion of the radiologist and was not protocolled. Reference standard ultrasound examinations were performed either by a radiological resident or by a staff radiologist. During office hours, ultrasound by residents was performed under the supervision of a staff radiologist.

\section{Computed tomography}

The CT parameters for the different CT systems in the original multicenter study were effective mAs level $165,120 \mathrm{kV},(4 \times)$ 2.5-mm collimation, $(4 \times) 3$-mm slice width and 0.5 -s rotation time, and $125 \mathrm{ml}$ iodinated contrast was given intravenously at $3 \mathrm{ml} / \mathrm{s}$ after a 60 -s delay. No orally or rectally administered contrast agents were used. Patients with known renal failure underwent non-enhanced CT. The CT images were evaluated in the same standardized manner as the ultrasound 
examination, except for transducer tenderness and compressibility of the appendix. A CT diagnosis was selected from the same predefined list of diagnoses, as used for the ultrasound diagnosis. The diagnosis of acute appendicitis was not protocolled and was left to the discretion of the reader who evaluated the images. Computed tomography was evaluated by a staff radiologist or by a resident under the supervision of a staff radiologist.

\section{Final diagnoses}

A final diagnosis was assigned to every patient by an expert panel, based on histopathology, imaging, and surgical findings, clinical information, and at least 6 months of followup. The panel consisted of two experienced gastrointestinal surgeons and an experienced abdominal radiologist. Every member of the expert panel individually evaluated all available evidence. In case of disagreement, consensus was achieved by group discussion. A final diagnosis of acute appendicitis was predominantly based on surgical findings, obtained histopathology, and follow-up data.

\section{Classification of diagnoses}

Diagnoses assigned by the expert panel, apart from acute appendicitis, were classified as urgent surgical conditions or as conditions not requiring urgent surgical intervention (urgent non-surgical conditions and non-urgent conditions). Urgent surgical conditions were defined as abdominal conditions requiring surgical intervention within $24 \mathrm{~h}$.

\section{Statistical analysis}

Only patients with a clinical suspicion of acute appendicitis, based on medical history, physical examination, and laboratory tests, were included in the analyses presented here. Estimates of sensitivity and specificity of imaging strategies for identification of acute appendicitis were calculated by comparing results of the imaging strategies against the final diagnoses, assigned by the expert panel, which served as the clinical reference standard. The results of the conditional CT strategy, as recommended by Dutch guidelines (http://www. nvgic.nl/richtlijnen/richtlijn\%20appendicitis.pdf), were based on ultrasound diagnoses in patients with an ultrasound examination positive for acute appendicitis and the CT diagnoses in the remaining patients: those with negative or inconclusive ultrasound findings. Subsequently, only CT diagnoses positive for acute appendicitis were labelled 'positive' while all other diagnoses were regarded 'inconclusive or negative'. For the results of the immediate CT strategy, the CT findings were used for all patients. Again, only CT diagnoses positive for acute appendicitis were labelled 'positive' while all other diagnoses were regarded 'inconclusive or negative'. We also calculated the percentage of missed cases of acute appendicitis, the number of false positives, and estimates of the positive predictive value and negative predictive value. In addition we calculated how many CTs could be avoided with a conditional CT strategy, by calculating the proportion of patients with positive ultrasound findings.

The difference in performance between the imaging strategies based on likelihood ratios were tested for statistical significance by considering ultrasound as 'triage test' as described by Macaskill et al $[13,14]$. Using this method, a direct comparison of positive and negative likelihood ratios is made. If one or both of the likelihood ratios do not improve significantly, there is no clear choice between the two strategies. All statistical analyses were performed with SPSS ${ }^{\circledR}$ software version 20.0 (IBM, Armonk, NY, USA). $P$ values of less than 0.05 were considered to indicate statistical significance.

\section{Results}

During a 19-month period, 1,101 consecutive patients presented with acute abdominal pain, 80 of whom had to be excluded because of incomplete case record forms [6]. Of the remaining 1,021 patients, 422 (41\%) had a clinical suspicion of acute appendicitis, based on medical history, physical examination, and laboratory tests. These were included in the present analysis. Their mean age was 40 years (range 19-89); $226(54 \%)$ were female. A list of the final diagnoses of these 422 patients, as assigned by the expert panel, is shown in Table 1. Acute appendicitis was the final diagnosis in 251 $(59 \%)$. Seventeen patients $(4.0 \%)$ had an alternate final diagnosis classified as an urgent surgical condition. The remaining 154 (36\%) patients were assigned final diagnoses classified as urgent non-surgical conditions or non-urgent conditions.

\section{Diagnostic accuracy for acute appendicitis}

Flow charts of the findings of the conditional CT strategy and the immediate CT strategy are depicted in Fig. 1A and B. In 199 patients (47\%) ultrasound imaging was inconclusive or negative for appendicitis. The estimated diagnostic accuracy of both strategies is shown in Table 2. The conditional CT strategy was able to identify correctly a similar number of patients with acute appendicitis, compared to the immediate CT strategy: 241 (96\%; $95 \% \mathrm{CI}, 93 \%$ to $98 \%$ ) versus 238 ( $95 \%$; $95 \%$ CI, $91 \%$ to $97 \%$ ) of 251 . However, the conditional CT strategy resulted in more false positives compared to the immediate CT strategy (39 versus 22 ), with a lower specificity of $77 \%$ (132 of $171 ; 95 \% \mathrm{CI}, 70 \%$ to $83 \%$ ) versus $87 \%$ (149 of $171 ; 95 \% \mathrm{CI}, 81 \%$ to $91 \%$ ) and a lower positive predictive value of $86 \%(95 \% \mathrm{CI}, 81 \%$ to $90 \%)$ versus $92 \%$ 
Table 1 Final diagnoses in 422 patients clinically suspected of having acute appendicitis

\begin{tabular}{|c|c|c|}
\hline Final diagnoses & $\mathrm{N}$ & $\%$ \\
\hline Acute appendicitis & 251 & 59 \\
\hline Other urgent surgical conditions & 17 & 4 \\
\hline Acute diverticulitis ${ }^{\mathrm{a}}$ & 6 & 1 \\
\hline Perforated viscus & 3 & 1 \\
\hline Adhesive small bowel obstruction & 2 & 0.5 \\
\hline Malignant bowel obstruction & 2 & 0.5 \\
\hline Ovarian torsion & 2 & 0.5 \\
\hline Acute cholecystitis & 1 & 0.2 \\
\hline Acute peritonitis & 1 & 0.2 \\
\hline Urgent non-surgical conditions & 19 & 5 \\
\hline Gynaecological diseases $^{\mathrm{b}}$ & 12 & 3 \\
\hline Retroperitoneal or abdominal wall bleeding & 3 & 1 \\
\hline Urological diseases $^{\mathrm{c}}$ & 3 & 1 \\
\hline Pneumonia & 1 & 0.2 \\
\hline Non-urgent conditions & 135 & 32 \\
\hline Nonspecific abdominal pain & 67 & 16 \\
\hline Acute diverticulitis ${ }^{\mathrm{d}}$ & 20 & 5 \\
\hline Inflammatory bowel disease & 18 & 4 \\
\hline Gastrointestinal diseases $^{\mathrm{e}}$ & 15 & 4 \\
\hline Urological diseases $^{\mathrm{f}}$ & 8 & 2 \\
\hline Malignancy & 2 & 0.5 \\
\hline Gynaecological diseases $^{\mathrm{g}}$ & 1 & 0.2 \\
\hline Other & 4 & 1 \\
\hline
\end{tabular}

a Acute diverticulitis with free air and/or free abdominal fluid on computed tomography

b Pelvic inflammatory disease, bleeding/ruptured ovarian cyst, tuboovarian abscess

${ }^{\mathrm{c}}$ Ureteral stones with obstruction, pyelonephritis

d Acute diverticulitis without free air or free abdominal fluid on computed tomography

e Gastroenteritis, acute appendagitis epiploica, cholecystolithiasis, obstipation

${ }^{\mathrm{f}}$ Renal and ureteral stones without obstruction, urinary tract infection

g Ovulation pain/bleeding

(95\%CI, $87 \%$ to $95 \%$ ). Comparison of the diagnostic performance of the direct CT and the conditional CT strategy based on likelihood ratios did not demonstrate superiority of one strategy.

Diagnostic accuracy for urgent surgical conditions

Imaging diagnoses compared against final diagnoses are listed in Table 3. Of the 39 patients with a false positive diagnosis of appendicitis following a conditional CT strategy, six had another underlying urgent surgical condition, compared to two of 22 after immediate CT imaging. The diagnostic accuracy of both imaging strategies in detecting urgent surgical conditions, including acute appendicitis, in patients with clinical suspicion of appendicitis is shown in Table 4. For urgent conditions, the conditional CT strategy performed comparably against the immediate $\mathrm{CT}$ strategy, with a sensitivity of $96 \%$ versus $94 \%$ and a negative predictive value of $92 \%$ versus $90 \%$. Here also, the specificity of the conditional strategy was significantly lower, $77 \%$ versus $85 \%$, as was the positive predictive value, with $88 \%$ versus $92 \%$. Evaluation of the diagnostic performances based on likelihood ratios revealed did not favour any strategy.

\section{Discussion}

In this study, an imaging strategy with conditional use of CT (CT only after inconclusive or negative ultrasound findings) was directly compared to an immediate CT strategy (CT in all) in adult patients with suspected acute appendicitis. The conditional CT strategy was able to identify correctly a similar number of appendicitis cases and more than halved the number of CT scans required. However, the conditional strategy resulted in more false positive cases of appendicitis. About one in five of these wrongly diagnosed patients had other underlying urgent surgical conditions, and the clinical consequences of these false positive diagnoses may, therefore, be limited. However, for the detection of urgent surgical conditions, including appendicitis, the conditional CT strategy still resulted in more false positives than the immediate $\mathrm{CT}$ strategy.

Our results confirm the ability of the conditional CT strategy to identify correctly patients with appendicitis. The higher rate of false positives in the conditional CT strategy compared to the immediate CT strategy is explained by the stepwise nature of the conditional CT strategy, since it accumulates positives of both modalities: both true and false positives. Hence, a conditional strategy will never have a lower sensitivity or a higher specificity than the unconditional, immediate strategy, by definition.

Patients considered to have appendicitis on CT after inconclusive or negative ultrasound were frequently false positives: 16 of the 57 patients $(28 \%)$ who had a positive result on CT after negative or inconclusive ultrasound findings did not have appendicitis as their final diagnosis. Apparently, both imaging modalities have limited diagnostic performance in these selected patients. The false positive rate of conclusive ultrasound was $10 \%$ (23 of 223), whereas CT in all patients resulted in $8 \%$ false positives ( 22 of 260 ). This good diagnostic performance of (conclusive) ultrasound, comparable to CT, suggests that more straightforward cases of appendicitis can adequately be detected by ultrasound.

Relatively high rates of false positives have previously been reported in a meta-analysis of head-to-head comparative studies evaluating ultrasound and $\mathrm{CT}$ for suspected 
Fig. 1 Flowchart of the results of imaging strategies with (A) conditional CT and (B) immediate CT in patients with clinical suspicion of acute appendicitis

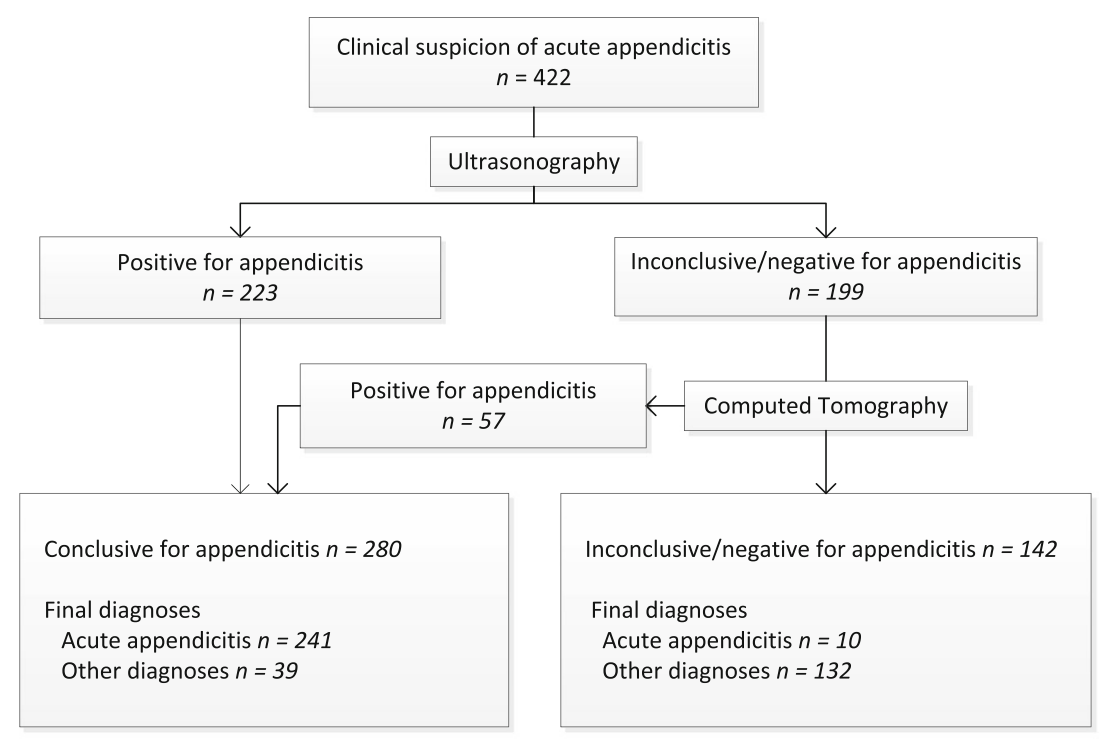

A Conditional CT

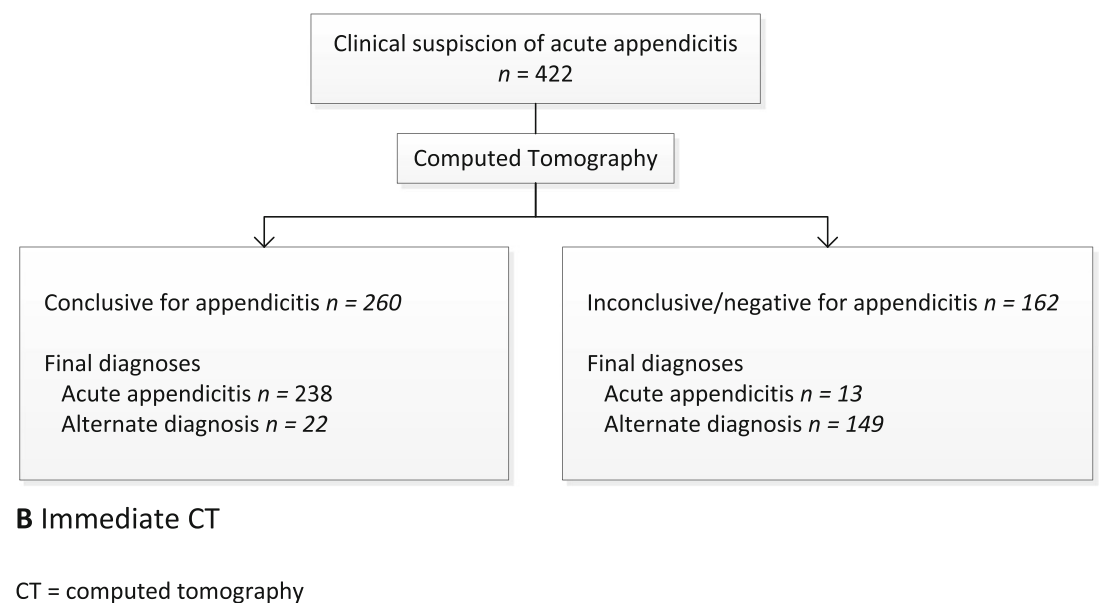

appendicitis [4]. The relatively high false positive rate of the conditional CT strategy is not reflected in reported rates of negative appendectomies after implementation of this strategy $[7,9,15,16]$. A possible explanation for this discrepancy is the fact that the classification of imaging results into positive or negative is an oversimplification of clinical practice. The level of certainty of the diagnosis is ignored while this may play an important role in clinical decision-making. The reported percentage of false positives may not directly lead to comparable percentages of negative appendectomies when the confidence level of the diagnosis is involved.

Similar differences in diagnostic performance were demonstrated for alternate surgical emergencies just as it was for acute appendicitis. In clinical practice, not only the detection of acute appendicitis is important, but also the correct identification of alternative underlying conditions in patients with clinical suspicion of acute appendicitis. Approximately half of the patients with an alternate final diagnosis are hospitalized

Table 2 Diagnostic accuracy of ultrasound with conditional CT versus immediate CT for the diagnosis of appendicitis in patients with clinical suspicion of acute appendicitis $(\mathrm{N}=422)$

\begin{tabular}{lllllll}
\hline Imaging strategy & Sensitivity & Missed cases & Specificity & Positive predictive value & False positive rate & Negative predictive value \\
\hline Conditional CT & $96 \%(93-98)$ & $4 \%$ & $77 \%(70-83)$ & $86 \%(81-90)$ & $14 \%$ & $93 \%(87-96)$ \\
& 241 of 251 & 10 of 251 & 132 of 171 & 241 of 280 & 39 of 280 & 132 of 142 \\
Immediate CT & $95 \%(91-97)$ & $5 \%$ & $87 \%(81-92)$ & $92 \%(87-95)$ & $8 \%$ & $92 \%(86-95)$ \\
& 238 of 251 & 13 of 251 & 149 of 171 & 238 of 260 & 22 of 260 & 149 of 162 \\
\hline
\end{tabular}

Values are percentages, numbers within parentheses indicate $95 \%$ confidence intervals. $\mathrm{CT}=$ computed tomography 
Table 3 Results of imaging strategies compared with final diagnoses as assigned by expert panel for appendicitis, other urgent surgical conditions and the remainder of diagnoses

\begin{tabular}{|c|c|c|c|}
\hline & \multicolumn{3}{|c|}{ Final diagnosis } \\
\hline & $\begin{array}{l}\text { Appendicitis } \\
(\mathrm{n}=251)\end{array}$ & $\begin{array}{l}\text { Other urgent surgical } \\
\text { condition }{ }^{\mathrm{a}} \\
(\mathrm{n}=17)\end{array}$ & $\begin{array}{l}\text { Urgent non-surgical } \\
\text { condition or non-urgent } \\
\text { condition } \\
(\mathrm{n}=154)\end{array}$ \\
\hline \multicolumn{4}{|l|}{ Conditional CT } \\
\hline Appendicitis & 241 & 6 & 33 \\
\hline Other urgent surgical condition ${ }^{\mathrm{a}}$ & 0 & 10 & 2 \\
\hline $\begin{array}{l}\text { Urgent non-surgical condition or non-urgent } \\
\text { condition } \\
\text { Immediate CT }\end{array}$ & 10 & 1 & 119 \\
\hline Appendicitis & 238 & 2 & 20 \\
\hline Other urgent surgical condition ${ }^{\mathrm{a}}$ & 0 & 13 & 3 \\
\hline Urgent non-surgical condition or non-urgent condition & 13 & 2 & 131 \\
\hline
\end{tabular}

${ }^{\text {a }}$ Other surgical urgent conditions were defined as conditions demanding surgical intervention within $24 \mathrm{~h}$. CT=computed tomography

and one in four undergo surgery or other invasive interventions [17].

To evaluate the diagnostic performance of the conditional CT strategy and the immediate CT strategy for alternate conditions, all diagnoses were categorized based on the need for surgical intervention; conditions demanding urgent surgical intervention (including acute appendicitis) and non-urgent conditions or urgent non-surgical conditions. Although the authors realize that this classification is arbitrary and represents an oversimplification of clinical practice, it does take into account the differences in management of various underlying conditions. The clinical consequence of falsely diagnosing a patient with appendicitis, and potentially commencing surgical treatment, while the patient has a simple urinary tract infection is different from falsely diagnosing a patient who has a perforated hollow viscus. When the imaging strategy is evaluated as a diagnostic test, both patients are considered to have false positive results. However, the latter patient would also need surgical treatment and the clinical consequences of the wrong diagnosis could, therefore, be limited.
The present study adds evidence to the debate whether a conditional CT strategy or an immediate CT strategy is preferred for adult patients with suspected acute appendicitis. Historically, a certain number of false positives and negative appendectomies is considered acceptable, to prevent missed cases of appendicitis with subsequent wrongful refrainment from treatment. Since the management of acute appendicitis is ever evolving, the desired characteristics of the preferred diagnostic test might change. For instance, with the potential conservative treatment of (uncomplicated) appendicitis, the consequences of false positive diagnoses would be overtreatment with antibiotics, instead of performing negative appendectomies [18].

Besides diagnostic performance, several other aspects should be taken into consideration when choosing the appropriate diagnostic strategy. Exposure to $\mathrm{CT}$ radiation with the risk of radiation-induced cancer is an increasing concern [5]. New techniques have made low-dose CT possible, and promising results are shown for diagnosing acute appendicitis [19, 20]. Either as a single test or as part of a conditional strategy,

Table 4 Diagnostic accuracy of ultrasound with conditional CT versus immediate CT for the diagnosis of all urgent surgical conditions (including acute appendicitis) in patients with clinical suspicion of acute appendicitis $(\mathrm{N}=422)$

\begin{tabular}{lllllll}
\hline Imaging strategy & Sensitivity & Missed cases & Specificity & Positive predictive value & False positive rate & Negative predictive value \\
\hline Conditional CT & $96 \%(93-98)$ & $4 \%$ & $77 \%(70-83)$ & $88 \%(84-91)$ & $12 \%$ & $92 \%(85-95)$ \\
& 257 of 268 & 11 of 268 & 119 of 154 & 257 of 292 & 35 of 292 & 119 of 130 \\
Immediate CT & $94 \%(91-97)$ & $6 \%$ & $85 \%(78-90)$ & $92 \%(88-95)$ & $8 \%$ & $90 \%(83-94)$ \\
& 253 of 268 & 15 of 268 & 131 of 154 & 253 of 276 & 23 of 276 & 131 of 146
\end{tabular}

Values are percentages; numbers within parentheses indicate $95 \%$ confidence intervals. Urgent surgical conditions were defined as conditions demanding surgical intervention within $24 \mathrm{~h}$. CT=computed tomography 
low-dose CT can potentially limit exposure to radiation without compromising diagnostic accuracy [21, 22]. The newest CT techniques even further decrease radiation dose. Alternative imaging modalities such as magnetic resonance imaging have also been studied and could potentially be implemented in diagnostic strategies for acute appendicitis [23]. An additional argument in the debate is the potential of the conditional strategy to offer medical costs savings [24]. A strategy based on ultrasound with selective use of CT, compared to only ultrasound or only $\mathrm{CT}$, was shown to be a costeffective imaging strategy in paediatric patients [25]. Furthermore, an increase in use of $\mathrm{CT}$ for acute appendicitis was demonstrated to increase cost of care, decrease contribution to margin, prolong patient's stay in the emergency department, and to delay time to operation [26].

Several potential limitations of this study need to be addressed. First, as stated previously, the classification of imaging results into positive or negative is an oversimplification of clinical practice. Furthermore, for all patients in whom the ultrasound diagnosis was not positive for appendicitis, the $\mathrm{CT}$ diagnosis was used for evaluating the diagnostic accuracy of the conditional CT strategy. In clinical practice, performing a CT is not always necessary if a certain diagnosis other than appendicitis is made on ultrasound. The performance of the conditional strategy in reducing the number of CTs needed could therefore be better than our reported results. In a study by Leeuwenburgh et al. the ultrasound examinations in 229 patients with suspected appendicitis were described [23]. In 11 patients, an urgent condition other than appendicitis was diagnosed on ultrasound. Therefore, omitting CT when certain diagnoses other than appendicitis are made on ultrasound would only further reduce the number of CT scans by approximately $5 \%$.

The ultrasound and CT examinations that are included in our study were performed in 2005 and 2006. Technological advances in $\mathrm{CT}$ and ultrasound imaging have been made during the last decade and have resulted in improved image quality. Potentially, diagnostic accuracy of both modalities for acute appendicitis has also improved. However, this improvement would likely be marginal and the relative difference in diagnostic accuracy between both modalities is presumably unaltered. Another potential limitation is the fact that when applying the conditional CT strategy in clinical practice, a CT after an inconclusive or negative ultrasound examination result is interpreted with knowledge of ultrasound findings. In our study, CTs after ultrasound were interpreted blinded from the ultrasound findings. We acknowledge that this study did not assess the effect of both strategies on clinical management and subsequent consequences. To address this issue, a study is needed comparing the clinical outcomes of patients suspected of having appendicitis randomized to diagnostic work-up according to either a conditional CT strategy or an immediate CT strategy. A final limitation of our study is the fact we did not evaluate all other aspects that should be taken into account when choosing the appropriate imaging strategy for suspected appendicitis. Our study merely illustrates the consequences of both studied strategies on pure diagnostic accuracy. As previously stated, several other aspects such as radiation exposure play an important role as well.

Our study represents the first head-to-head comparison of two frequently applied imaging strategies in adult patients with clinical suspicion of acute appendicitis. This direct comparison avoids the introduced bias when two imaging modalities are compared among different populations. The appendicitis prevalence was $57 \%$, which falls within the range of previously reported prevalence rates in other studies including patients who are clinically suspected of having acute appendicitis $[4,27]$. The prevalence of the disease under study is known to influence the diagnostic performance of a diagnostic test [4].

When choosing the appropriate imaging strategy for suspected appendicitis, the advantage of the conditional CT strategy (half the number of CTs needed) should be weighed against the potential clinical consequence of the increase in false positive diagnoses.

Acknowledgments The scientific guarantor of this publication is M.A. Boermeester. The authors of this manuscript declare no relationships with any companies, whose products or services may be related to the subject matter of the article. This study has received funding by The Dutch Organization for Health Research and Development (ZonMW) as part of the Health Care Efficiency Research program (ZonMW grant number: 94504-308). One of the authors has significant statistical expertise (P.M. Bossuyt). Institutional review board approval was obtained. Written informed consent was obtained from all subjects (patients) in this study. Some study subjects or cohorts have been previously reported in: Lameris W, van Randen A, van Es HW et al. Imaging strategies for detection of urgent conditions in patients with acute abdominal pain: diagnostic accuracy study. BMJ 2009; 338:b2431.

Methodology: prospective, diagnostic study, multicenter study.

Open Access This article is distributed under the terms of the Creative Commons Attribution Noncommercial License which permits any noncommercial use, distribution, and reproduction in any medium, provided the original author(s) and the source are credited.

\section{References}

1. Cuschieri J, Florence M, Flum DR et al (2008) Negative appendectomy and imaging accuracy in the Washington State Surgical Care and Outcomes Assessment Program. Ann Surg 248:557-563

2. Drake FT, Florence MG, Johnson MG et al (2012) Progress in the diagnosis of appendicitis: a report from Washington State's Surgical Care and Outcomes Assessment Program. Ann Surg 256:586-594

3. Terasawa T, Blackmore CC, Bent S, Kohlwes RJ (2004) Systematic review: computed tomography and ultrasonography to detect acute appendicitis in adults and adolescents. Ann Intern Med 141:537-546

4. van Randen A, Bipat S, Zwinderman AH et al (2008) Acute appendicitis: meta-analysis of diagnostic performance of CT and graded compression US related to prevalence of disease. Radiology 249: 97-106 
5. Brenner DJ, Hall EJ (2007) Computed tomography-an increasing source of radiation exposure. N Engl J Med 357:2277-2284

6. Lameris W, van Randen A, van Es HW et al (2009) Imaging strategies for detection of urgent conditions in patients with acute abdominal pain: diagnostic accuracy study. BMJ 338:b2431

7. Poortman P, Oostvogel HJ, Bosma E et al (2009) Improving diagnosis of acute appendicitis: results of a diagnostic pathway with standard use of ultrasonography followed by selective use of CT. J Am Coll Surg 208:434-441

8. van Breda Vriesman AC, Kole BJ, Puylaert JB (2003) Effect of ultrasonography and optional computed tomography on the outcome of appendectomy. Eur Radiol 13:2278-2282

9. Leeuwenburgh MM, Bakker OJ, Gorzeman MP et al (2010) Fewer unnecessary appendectomies following ultrasonography and CT. Ned Tijdschr Geneeskd 154:A869

10. Coursey CA, Nelson RC, Patel MB et al (2010) Making the diagnosis of acute appendicitis: do more preoperative CT scans mean fewer negative appendectomies? A 10-year study. Radiology 254:460-468

11. Raja AS, Wright C, Sodickson AD et al (2010) Negative appendectomy rate in the era of CT: an 18-year perspective. Radiology 256 : 460-465

12. Lameris W, van RA, Dijkgraaf MG et al (2007) Optimization of diagnostic imaging use in patients with acute abdominal pain (OPTIMA): design and rationale. BMC Emerg Med 7:9

13. Hayen A, Macaskill P, Irwig L, Bossuyt P (2010) Appropriate statistical methods are required to assess diagnostic tests for replacement, add-on, and triage. J Clin Epidemiol 63:883-891

14. Macaskill P, Walter SD, Irwig L, Franco EL (2002) Assessing the gain in diagnostic performance when combining two diagnostic tests. Stat Med 21:2527-2546

15. Krishnamoorthi R, Ramarajan N, Wang NE et al (2011) Effectiveness of a staged US and CT protocol for the diagnosis of pediatric appendicitis: reducing radiation exposure in the age of ALARA. Radiology 259:231-239

16. Toorenvliet BR, Wiersma F, Bakker RF et al (2010) Routine ultrasound and limited computed tomography for the diagnosis of acute appendicitis. World J Surg 34:2278-2285
17. Pooler BD, Lawrence EM, Pickhardt PJ (2012) Alternative diagnoses to suspected appendicitis at CT. Radiology 265:733-742

18. Varadhan KK, Neal KR, Lobo DN (2012) Safety and efficacy of antibiotics compared with appendicectomy for treatment of uncomplicated acute appendicitis: meta-analysis of randomised controlled trials. BMJ 344:e2156

19. Kim SH, Yoon JH, Lee JH et al. (2014) Low-dose CT for patients with clinically suspected acute appendicitis: optimal strength of sinogram affirmed iterative reconstruction for image quality and diagnostic performance. Acta Radiol

20. Willemink MJ, Leiner T, de Jong PA et al (2013) Iterative reconstruction techniques for computed tomography part 2: initial results in dose reduction and image quality. Eur Radiol 23:1632-1642

21. Kim K, Kim YH, Kim SY et al (2012) Low-dose abdominal CT for evaluating suspected appendicitis. N Engl J Med 366:1596-1605

22. Poletti PA, Platon A, De PT et al (2011) Acute appendicitis: prospective evaluation of a diagnostic algorithm integrating ultrasound and low-dose CT to reduce the need of standard CT. Eur Radiol 21:2558 2566

23. Leeuwenburgh MM, Wiarda BM, Wiezer MJ et al (2013) Comparison of imaging strategies with conditional contrastenhanced CT and unenhanced MR imaging in patients suspected of having appendicitis: a multicenter diagnostic performance study. Radiology 268:135-143

24. Parker L, Nazarian LN, Gingold EL et al (2014) Cost and radiation savings of partial substitution of ultrasound for CT in appendicitis evaluation: a national projection. AJR Am J Roentgenol 202:124 135

25. Wan MJ, Krahn M, Ungar WJ et al (2009) Acute appendicitis in young children: cost-effectiveness of US versus CT in diagnosis-a Markov decision analytic model. Radiology 250:378-386

26. Pritchett CV, Levinsky NC, Ha YP, Dembe AE, Steinberg SM (2010) Management of acute appendicitis: the impact of CT scanning on the bottom line. J Am Coll Surg 210:699-705

27. Andersson RE (2004) Meta-analysis of the clinical and laboratory diagnosis of appendicitis. Br J Surg 91:28-37 